\title{
A special issue on NF-KB signaling and function
}

\author{
Cell Research (2011) 21:1-2. doi:10.1038/cr.2011.1; published online 3 January 2011
}

Guest editors

Shao-Cong Sun ${ }^{1}$

${ }^{1}$ Department of Immunology,

The University of Texas MD

Anderson Cancer Center and

The University of Texas Graduate School of Biomedical Sciences at Houston, 7455 Fannin

Street, Box 902, Houston TX

77030, USA

ssun@mdanderson.org

Zheng-Gang $\mathrm{Liu}^{2}$

${ }^{2}$ Cell and Cancer Biology

Branch, Center for Cancer

Research, National Cancer

Institute, National Institutes of

Health, Bethesda, MD, USA

zgliu@box-z.nih.gov
$S_{\text {ince its discovery about } 25 \text { years ago, }}$ the NF- $\kappa B$ signaling pathway has remained one of the exciting and extensively studied fields of biomedical research. It is of no doubt that NF- $\mathrm{KB}$ functions as an essential intracellular messenger that connects various environmental signals to the induction of numerous cellular genes. Not surprisingly, NF$\kappa \mathrm{B}$ regulates diverse biological processes, ranging from cell growth and survival, tissue and organ development to immune response and inflammation. Deregulated NF- $\kappa B$ signaling is associated with many important human diseases, such as cancer, autoimmunity, chronic inflammation, metabolic disorders, and neurodegenerative diseases. Therefore, understanding the molecular mechanisms that regulate $\mathrm{NF}-\mathrm{\kappa B}$ signaling and function is important for exploiting this cellular pathway in therapeutic approaches to treat human diseases. This special issue of Cell Research presents a series of review articles that discuss our current understanding of $\mathrm{NF}-\mathrm{\kappa B}$ regulation and function and its role in some human diseases.

Protein ubiquitination has become a wellaccepted mechanism of NF- $\mathrm{\kappa B}$ regulation, which functions through both proteasomal degradation of NF- $\mathrm{KB}$ inhibitors and catalytic activation of IкB kinase (IKK) and its upstream signaling components (reviewed by Liu \& Chen). Of particular interest is the lysine (K) 63 polyubiquitin chains, which facilitate the assembly of IKK signaling complexes and probably are also directly engaged in the activation of IKK or its upstream kinases. Like protein phosphorylation, protein ubiquitination is a reversible process that is counter-regulated by ubiquitin ligases and deubiquitinases (DUBs). The proper function of NF- $\mathrm{KB}$ requires its negative regulation by specific DUBs, which act by terminating receptor-induced NF- $\kappa \mathrm{B}$ signaling or preventing spontaneous activation of NF- $\mathrm{KB}$ (reviewed by Harhaj \& Dixit). Another biochemical mechanism of NF- $\mathrm{KB}$ regulation is proteolysis. In addition to the proteasome, the capase family of proteases participates in the regulation of NF- $\mathrm{KB}$ signaling downstream of specific receptors. Beyaert and colleagues discuss how caspases and the paracaspase MALT1 modulate NF- $\mathrm{kB}$ signaling and thereby regulate inflammation versus apoptosis.

A remarkable feature of NF- $\mathrm{KB}$ signaling is its triggering by a large variety of receptors. How the NF- $\mathrm{kB}$ pathway is connected to the different signaling receptors has been an active area of research. Studies over the past two decades have led to the discovery of specific adaptors and scaffold proteins involved in receptor-specific NF- $\mathrm{kB}$ activation. In particular, the CARMA family of scaffold proteins mediates NF- $\mathrm{kB}$ activation by various receptors, including the antigen receptors, Fc receptors, G protein-coupled receptors, and C-type lectin recptors, thereby regulating immunity and oncogenesis (reviewed by Blonska and Lin).

In addition to the canonical (typical) pathway of NF- $\mathrm{KB}$ activation, there are atypical pathways that regulate more specific aspects of NF- $\mathrm{KB}$ signaling. An important and extensively studied atypical pathway is the so-called noncanonical NF- $\kappa \mathrm{B}$ pathway, which relies on the processing of the NF- $\kappa B$ precursor protein p100 (reviewed by Sun). This pathway differs from the canonical NF$\kappa B$ pathway in both the signaling mechanisms and the eliciting receptors. In contrast to the diverse functions of the canonical NF- $\mathrm{KB}$ pathway, the noncanonical NF- $\kappa B$ pathway regulates specific biological processes, such as immune cell maturation and differentiation and lymphoid organogenesis. However, it is 
important to note that the canonical and noncanonical NF- $\kappa \mathrm{B}$ pathways have extensive crosstalks and thus should be viewed as different axes of the same signaling system (reviewed by Shih et al.).

Originally discovered as a transcription factor regulating immunoglobulin gene expression, $\mathrm{NF}-\kappa \mathrm{B}$ has now been assigned with numerous physiological and pathological functions. One important function of NF- $\kappa \mathrm{B}$ is to respond to stress signals. Morgan and Liu discuss how the NF- $\kappa \mathrm{B}$ pathway is influenced by reactive oxygen species (ROS), and conversely how the regulation of pro- or anti- oxidant genes by NF- $\mathrm{KB}$ influences the production or mitigation of ROS. Crosstalk between ROS and NF- $\kappa \mathrm{B}$ is important in maintaining the appropriate cellular levels of ROS, preventing cellular damage, and generally promoting cell survival during oxidative stress. NF- $\kappa \mathrm{B}$ also responds to genotoxic agents that cause cellular stress by damaging chromosomal DNA. This type of stress response is mediated by a nucleus-initiated signaling pathway that involves NEMO modification and subsequent IKK activation by the DNA damage-responsive kinase ATM (ataxia telangiectasia mutated) (reviewed by Miyamoto).

Probably the best-defined function of $\mathrm{NF}-\kappa \mathrm{B}$ is to regulate the development and activation of the immune system. $\mathrm{NF}-\kappa \mathrm{B}$ is essential for proper function of both the innate and adaptive immune systems. The review by Hayden and Ghosh focuses on the recent progress in this rapidly developing area. In another review, Pasparakis and colleagues discuss how NF- $\kappa \mathrm{B}$ regulates immune homeostasis and inflammation in the intestine and skin, major natural barriers of the host defense system. Remarkably, too much or too little NF- $\kappa \mathrm{B}$ activity can lead to chronic inflammation in these tissues. The review by Ley and colleagues points to the complexity of $\mathrm{NF}-\kappa \mathrm{B}$ function in the immune system. In addition to directly transactivating immune and inflammatory genes, NF- $\mathrm{B}$ acts through crosstalk with other signaling pathways. The authors discuss how IKK and an NF- $\kappa \mathrm{B}$ precursor protein, $\mathrm{p} 105$, regulate $\mathrm{Tpl}-2$, thereby controlling the activation of ERK MAP kinase pathway in innate immune cells.

Consistent with the function of $\mathrm{NF}-\kappa \mathrm{B}$ in regulating cell growth and survival, deregulated NF- $\kappa \mathrm{B}$ activation is linked to oncogenesis. It is now a well-known phenomenon that the $\mathrm{NF}-\kappa \mathrm{B}$ signaling pathway is constitutively activated in various cancer cells. However, how NF- $\kappa \mathrm{B}$ regulates cancer development remains incompletely understood and, in some cases, even largely controversial. The review by He and Karin proposes a model for how NF- $\mathrm{B}$ promotes liver cancer development, suggesting the involvement of STAT3 activation by the NF- $\kappa$ Bregulated cytokine IL-6. The authors also discuss how NF- $\mathrm{B}$ may, under certain conditions, function to inhibit liver cancer development as revealed in some animal studies and point out the limitations of currently available animal models for the study of human hepatocellular carcinoma.

The review by Novack describes the role of $\mathrm{NF}-\kappa \mathrm{B}$ in regulating bone homeostasis, another actively studied area of $\mathrm{NF}-\kappa \mathrm{B}$ research. This function of NF- $\mathrm{B}$ involves both the canonical and noncanonical pathways, which act on different types of cells in the bone system. The author also discusses how deregulated NF- $\kappa \mathrm{B}$ activation contributes to the development of various disease states of the bone.

A unique and powerful approach for $\mathrm{NF}-\kappa \mathrm{B}$ investigation is structural analysis, which provides a clearer picture about how the activation and function of NF- $\kappa \mathrm{B}$ signaling components are regulated at the molecular level. Wu and colleagues describe the recent structural discoveries in the NF- $\kappa \mathrm{B}$ pathway and address a number of important questions regarding the activation, function, and feedback inhibition of NF- $\kappa$ B.

In summary, this special issue covers a broad, although not every, aspect of $\mathrm{NF}-\kappa \mathrm{B}$ signaling and functions. We sincerely hope that the review articles presented here will offer the readers sufficient introductory knowledge as well as current information of this fascinating field of biomedical research. 